\title{
PRELIMINARY TOPOGRAPHIC DIAGNOSIS OF ISCHEMIC BRAIN INJURIES ACCORDING TO SPEECH AND LANGUAGE DISORDERS (ABSTRACT)*. DISSERTATION. CAMPINAS, 2009.
}

\section{FABRICIO FERREIRA DE OLIVEIRA**}

Introduction: Topographic diagnosis of ischemic brain injuries depends on signs and symptoms presented by patients, which may be restricted to speech and language.
Objective: In such cases, proper evaluation may predict lesion topography and guide therapeutic measures.

Method: A non-standardized battery was employed for 
evaluation of aphasia and dysarthria in the acute stroke phase, consisting on tests for attentional deficits, handedness, visual perception, phonemic perception, spontaneous speech, fluency, comprehension, word and sentence repetition, naming, automatic speech, ideomotor praxis and constructional praxis. Recruitment was carried out for 12 months (May 2007 to April 2008) at the Hospital das Clínicas - UNICAMP, initially with the enrollment of ischemic stroke patients at the Emergency Unit. Thirty-seven first-stroke patients ( 22 male, 15 female) were submitted to the test in the first 72 hours after the brain injury, with the following additional inclusion criteria : adults over 18 years-old, any educational level, not comatose, no evidence of previous strokes or other brain diseases, no decompensated systemic diseases, no history of chronic alcoholism or neurotoxic substance abuse. The examiner was blind to stroke topography during the query. Subjects were paired with thirty-seven healthy controls according to age ( \pm 5 years) and schooling ( \pm 2 years). All patients underwent radiological evaluation (cerebral computed tomography and/or magnetic resonance) for topographic correlation between speech and language assessment results and the brain injury site, both in the acute and the chronic stroke phases. In statistical analysis, either chisquare test was employed for comparison of categorical variables or Fisher's exact test when necessary; for continuous measures to be ordered in two groups the Mann-Whitney test was used; ANOVA was applied for comparisons among three or more groups, while Tukey's test was employed for multiple comparisons. For identification of variables with the capacity to discriminate lesion side (left or right), multiple logistic regression analysis was used. The threshold of significance was set at $p<0.05$. All survivors were followed for a period of 3-18 months after the acute stroke, in order to be evaluated for the evolution of their speech and/or language disorders.

Results: Overall, classic clinical-anatomic correlations were found pertaining brain injury topography in relation to the aphasia subtype presented by each subject, with two exceptions. Since there were no patients with conduction aphasia, anomic aphasia, non-thalamic subcortical aphasia or apraxia of speech in our sample, these specific forms of speech and language impairment could not be evaluated. All test items were considered of significance for comparisons between the linguistic evaluation and the brain injury topography for stroke patients, except for age and schooling, as expected. Handedness, age and schooling were similar for groups of patients and controls, with no statistically significant difference. Subjects with classical aphasia subtypes and/or dysarthria had significantly more speech and language dysfunction in all assessed items than healthy controls.

Conclusion: Neurolinguistic examination may accurately predict the vascular territories involved in ischemic brain injuries, considering speech and/or language disturbances presented, patient history and neurological examination, as well as neuroimaging exams for comparison. Global aphasia and lone cortical dysarthria carried a worse prognosis for language and speech evolution. Brain injury size was the most influent factor for linguistic recovery at 3 months post-stroke. The most important tests for discrimination of brain injury sides (left or right) were: word and sentence repetition, naming and ideomotor praxis tests, as well as the comprehension tests in a minor degree of significance.

Key words: linguistics, aphasia, stroke, brain infarction, language, speech, disability evaluation, prognosis.

\footnotetext{
*Diagnóstico localizatório preliminar da lesão vascular cerebral isquêmica com base em distúrbios de fala e linguagem (Resumo). Dissertação de Mestrado. Faculdade de Ciências Médicas - Universidade Estadual de Campinas (UNICAMP) - Campinas/SP (Área: Ciências Médicas - Concentração em Neurologia). Orientador: Benito Pereira Damasceno. Financiamento: CAPES - Coordenação de Aperfeiçoamento de Pessoal de Nivel Superior.
}

**Address: Rua Albano de Almeida Lima 311 - 13073-131 Campinas SP - Brasil (E-mail: fabricioferreiradeoliveira@hotmail.com). 